\title{
INVESTIGATION OF OFFSET RECOVERY ALGORITHMS FOR HIGH PERFORMANCE BLUETOOTH RECEIVERS
}

\author{
Charles Tibenderana and Stephan Weiss \\ Communications Research Group, School of Electronics and Computer Science \\ University of Southampton, Southampton SO17 1BJ, UK
}

Keywords: Bluetooth, synchronisation, carrier offser, modulation index offset.

\begin{abstract}
Research has shown that significant degradation in performance can occur even when operating within the limits for carrier frequency and modulation index offsets permitted by the Bluetooth standard. This is even more severe for multi-bit detectors like the matched filter bank receiver, because such errors accumulate across a longer observation interval. This paper details the derivation of a stochastic gradient carrier frequency offset correction algorithm suitable for Bluetooth signals, as well as a separate algorithm applicable to the efficient realisation of the matched filter bank (MFB) receiver for Bluetooth signals. The reduced complexity MFB receiver for Bluetooth is described in our earlier publications.
\end{abstract}

\section{Introduction}

This paper extends research carried out at the University of Southampton to build a Bluetooth receiver for software defined radio (SDR) that has a high BER performance, yet is as efficient as possible in terms if complexity, and is robust to common signal adversities. Already we have proposed a new technique to reduce the complexity of a high-performance matched filter bank (MFB) receiver [1, 2, 3] for Bluetooth signals by up to $80 \%[4,5,6]$, and suggested a way to provide speedy equalisation despite correlated transmit signal samples and the possibility of significant carrier frequency offsets between the transmitter and receiver [7].

However, other researchers have established that carrier frequency offsets of the magnitude permitted by the Bluetooth standard [8] can degrade the performance of a single-bit detection algorithms [9], and this will be more severe for multibit detectors like the MFB receiver, in which carrier frequency errors accumulate across a longer observation interval [10]. Hence, we introduced a stochastic gradient (SG) [11] carrier frequency compensation procedure suitable for Bluetooth in [4, 12], and in [13] we put forward a separate carrier frequency synchronisation method that is based on intermediate filter outputs (IFO) that are inherent with the low-complexity realisation of the MFB receiver.

In this paper we present a detailed derivation of the synchronisation methods mentioned above, because such a complete mathematical analysis has never been published. To accomplish this, this paper is structured as follows: After this in- troduction, a signal model is developed in Sec. 2, leading to the formulation of the SG carrier frequency correction algorithm in Sec. 3. The low-complexity realisation of the MFB receiver is reviewed in Sec. 4.1, paving the way to derive the IFO carrier frequency synchronisation procedure in Sec. 4.2, and extend the discussion to synchronisation of modulation index in Sec. 4.3. Selected results appear in Sec. 5, before concluding in Sec. 6.

\section{Signal Model}

The modulation method specified for Bluetooth is Gaussian frequency shift keying (GFSK) [8], which generally involves the frequency modulation (FM) of a multilevel symbol $p[k]$, assumed here to be binary, $p[k] \in\{ \pm 1\}$. This bit sequence is expanded by a factor of $N$ and passed through a Gaussian filter with impulse response $g[n]$ of length $L N$, thus having a support of $L$ bit periods and yielding a continuous instantaneous angular frequency signal

$$
\omega[n]=2 \pi h \sum_{k=-\infty}^{\infty} p[k] g[n-k N],
$$

where $h$ is the modulation index. The phase of the analytic baseband transmitted signal,

$$
s[n]=\exp \left\{j \sum_{\nu=-\infty}^{n} \omega[\nu]\right\}=\prod_{\nu=-\infty}^{n} e^{j \omega[\nu]},
$$

is determined as the cumulative sum over all previous phase values $\omega[n]$.

We assume that the transmitted signal is subjected to a carrier frequency offset $\Delta \Omega$, a gain $A$, and additive white Gaussian noise (AWGN) $v[n]$ that is uncorrelated with the transmitted signal $s[n]$, such that the received signal is defined as

$$
r[n]=A \cdot s[n] e^{j \Delta \Omega n}+v[n] .
$$

In (3) the term $\Delta \Omega$ refers to the normalised angular carrier frequency offset, and is related to the carrier frequency offset $\Delta f \mathrm{~Hz}$, by the expression

$$
\Delta \Omega=\frac{2 \pi \Delta f}{N R},
$$

where $R$ symbolises the data rate. The signal development is summarised in Fig. 1. 


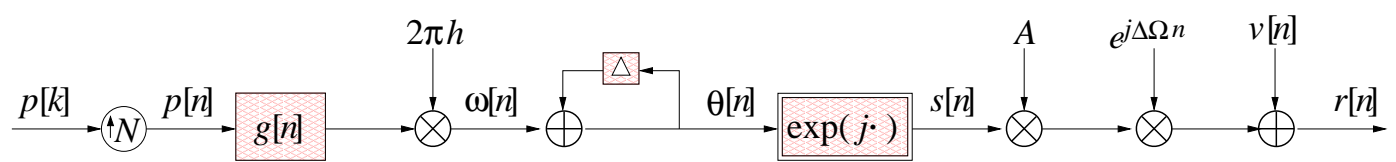

Fig. 1. Signal model.

\section{Stochastic Gradient Carrier Frequency Offset Correction Algorithm}

\subsection{Detection}

An estimation of the carrier frequency offset can be based on the received signal in (3), as shown in [6], by denoting

$$
\begin{aligned}
\mathcal{E}\left\{r[n] r^{*}[n-M]\right\} & =\left(A \mathcal{E}\{s[n]\} e^{j \Delta \Omega n}+\mathcal{E}\{v[n]\}\right) \\
& =|A|^{2} e^{j \Delta \Omega M}
\end{aligned}
$$

where $\mathcal{E}\{\cdot\}$ is the expectation operator and $M$ is a positive integral delay constant; the result in (4) rests on the assumptions of independence and zero mean of $s[n]$ and $v[n]$. Since the instantaneous frequency accumulated over $M$ samples of the transmitted signal $s[n]$ will either rotate in a positive or negative direction but on average be zero, we have $\mathcal{E}\left\{s[n] s^{*}[n-M]\right\}=$ 1 , while selecting $M$ sufficiently large ensures that the noise autocorrelation term vanishes, thus enabling the simplification in (4).

\subsection{Cost Function}

We create a modified receiver input $\tilde{r}[n]$,

$$
\tilde{r}[n]=\operatorname{Br}[n] e^{j \Theta n},
$$

i.e. modulating by $\Theta$ and scaling the input by $B$, which is ideally selected such that $B^{-1}=|r[n]|=A$, and $\Theta$ to match

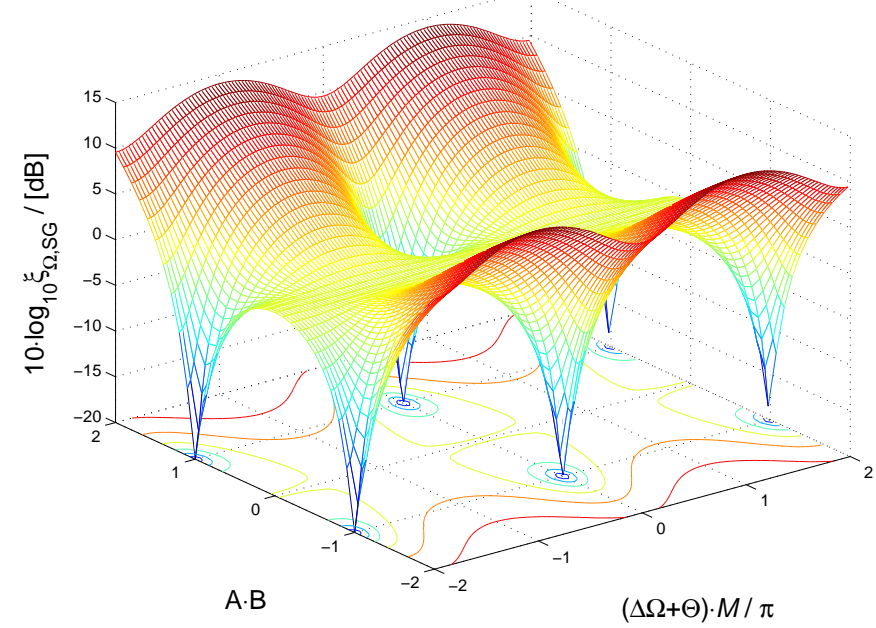

Fig. 2. Cost function for the SG carrier frequency offset correction algorithm $\xi_{\Omega, \mathrm{SG}}$ in (7). the carrier offset $\Delta \Omega$. In order to determine $\Theta$, we can use the following constant modulus (CM) cost function,

$$
\xi_{\Omega, \mathrm{SG}}=\left|\mathcal{E}\left\{\tilde{r}[n] \tilde{r}^{*}[n-M]\right\}-1\right|^{2} .
$$

Inserting (5) and (4) into (6) yields the cost function shown in Fig. 2, which is given by

$$
\begin{aligned}
\xi_{\Omega, \mathrm{SG}} & =\left(\mathcal{E}\left\{\tilde{r}[n] \tilde{r}^{*}[n-M]\right\}-1\right)\left(\mathcal{E}\left\{\tilde{r}^{*}[n] \tilde{r}[n-M]\right\}-1\right) \\
& =1+|A B|^{4}-2|A B|^{2} \cos ((\Theta+\Delta \Omega) M),
\end{aligned}
$$

as derived in [6] with

$$
\xi_{\Omega, \mathrm{SG}}=0 \longleftrightarrow \Theta=\frac{2 \pi k}{M}-\Delta \Omega \quad \vee \quad|A B|=1 .
$$

We are interested in the solution for $k=0$ only, for which the cost function provides a unique minimum under the condition

$$
-\pi<(\Theta+\Delta \Omega) M<\pi
$$

\subsection{Stochastic Gradient Method}

Within the bounds of (8), $\Theta$ can be iteratively adapted over time based on gradient descend techniques according to

$$
\Theta[n+1]=\Theta[n]-\mu_{\Theta} \frac{\partial \xi_{\Omega, \mathrm{SG}}}{\partial \Theta}
$$

with a suitable step size parameter $\mu_{\Theta}$. A stochastic gradient can be based on an instantaneous cost $\hat{\xi}_{\Omega \text {,SG }}$ by omitting expectations in (6) and assuming small changes in $\Theta$ only, it can be shown [6] that

$$
\begin{aligned}
& \frac{\partial \hat{\xi}_{\Omega, \mathrm{SG}}[n]}{\partial \Theta}= \frac{\partial}{\partial \Theta}\left\{\left(B^{2} r[n] r^{*}[n-M] e^{j \Theta M}-1\right)\right. \\
&=\left.\left(B^{2} r^{*}[n] r[n-M] e^{-j \Theta M}-1\right)\right\} \\
&=\left(\tilde { I } \operatorname { I m } \left\{\tilde{r}[n] \tilde{r}^{*}[n-M] \cdot\right.\right. \\
&\left.\left.\cdot(n] \tilde{r}^{*}[n-M]-1\right)^{*}\right\}
\end{aligned}
$$

Similarly, the gain parameter $B$ in (5) can be adjusted by

$$
B[n+1]=B[n]-\mu_{B} \frac{\partial \xi_{\Omega, \mathrm{SG}}}{\partial B}
$$

whereby the stochastic gradient can derived analogously to above the results as [6]

$$
\begin{aligned}
& \frac{\partial \hat{\xi}_{\Omega, \mathrm{SG}}[n]}{\partial B}=\frac{\partial}{\partial B}\left\{\left(B^{2} r[n] r^{*}[n-M] e^{j \Theta M}-1\right)\right.\left.\cdot\left(B^{2} r^{*}[n] r[n-M] e^{-j \Theta M}-1\right)\right\} \\
&=\frac{4}{B} \operatorname{Re}\left\{\tilde{r}[n] \tilde{r}^{*}[n-M] \cdot\right. \\
&\left.\cdot\left(\tilde{r}[n] \tilde{r}^{*}[n-M]-1\right)^{*}\right\}
\end{aligned}
$$




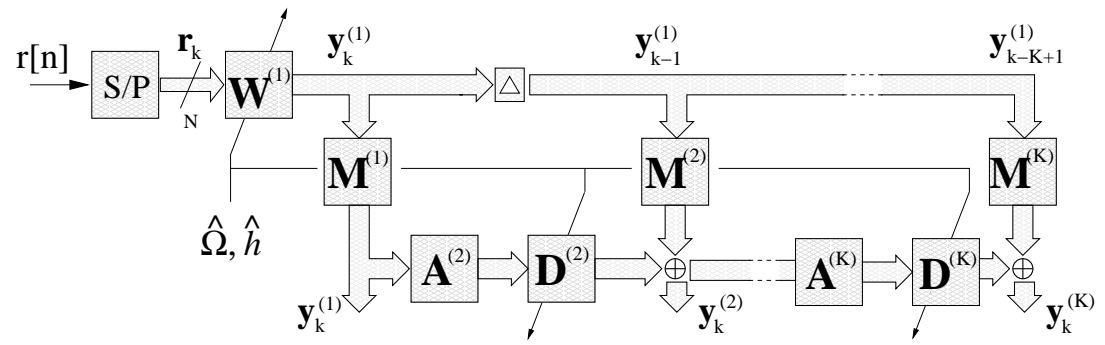

Fig. 3. Low-complexity implementation of a matched filter bank high-performance GFSK receiver. The received GFSK signal $r[n]$ is passed through a serial/parallel converter and a smaller intermediate filter bank $\mathbf{W}^{(1)}$ with a single symbol duration. Processed over $K$ stages, the matched filter bank outputs are contained in $\mathbf{y}_{k}^{(K)}$.
Instead of $r[n]$, the modified received signal $\tilde{r}[n]$ in (5) would then be passed into the matched filter detector discussed in Sec. 4.1.

\section{Intermediate Filter Output Based Offset Cor- rection}

\subsection{Efficient MFB Receiver}

The low-complexity matched filter bank receiver for Bluetooth signals is portrayed in Fig. 3, however only a summary of its derivation in $[4,5,6]$ shall he highlighted in the following. Simply speaking, the efficient realisation of the MFB eliminates redundancy in providing the matched filter responses for a filter bank $\mathbf{W}^{(K)}$ that is $K$-bit periods in length, by employing a relatively small 1-bit period long intermediate filter bank $\mathbf{W}^{(1)}$. Intermediate filter outputs for $K$ bit periods are stored and processing appropriately to obtain the results for $\mathbf{W}^{(K)}$.

For example, the MFB outputs over a single bit period, $K=1$, can be calculated as

$$
\mathbf{y}_{k}^{(1)}=\mathbf{W}^{(1)} \mathbf{r}_{k}
$$

where the column vector $\mathbf{r}_{k}$ represents the received signal samples $r[n]$ held in a length $N$ tap delay line, and $\mathbf{W}^{(1)} \in \mathbb{C}^{2^{L} \times N}$ contains the $2^{L}$ legitimate matching signals $s^{*}[-n]$ in its rows, and represents an intermediate filter bank. In the case where $L=3$, the phase trajectories of the resulting 8 waveforms are shown in Fig. 4. The superscript ${ }^{(1)}$ in (11) indicates that only a single bit period $K=1$ is observed.

For $K>1$, the possible phase trajectories can be derived from the case $K-1$ by augmentation of the curves shown in Fig. 4 and the application of appropriate phase shifts. This allows us to formulate iteratively for $\mathbf{y}_{k}^{(K)} \in \mathbb{C}^{2^{K+L-1}}[4,5,6]$

$$
\mathbf{y}_{k}^{(K)}=\mathbf{D}^{(K)} \mathbf{A}^{(K)} \mathbf{y}_{k}^{(K-1)}+\mathbf{M}^{(K)} \mathbf{y}_{k-K+1}^{(1)},
$$

where

$$
\begin{aligned}
& \mathbf{M}^{(K)}=\left[\begin{array}{l}
\mathbf{M}^{(K-1)} \\
\mathbf{M}^{(K-1)}
\end{array}\right] \quad \text { with } \quad \mathbf{M}^{(1)}=\mathbf{I}_{2^{L}} \\
& \mathbf{A}^{(K)=}\left[\begin{array}{lr}
\mathbf{A}^{(K-1)} & \mathbf{0} \\
\mathbf{0} & \mathbf{A}^{(K-1)}
\end{array}\right] \quad \text { with } \\
& \mathbf{A}^{(1)}=\text { blockdiag }\left\{\left[\begin{array}{l}
1 \\
1
\end{array}\right]\right\} \in \mathbb{Z}^{2^{L} \times 2^{L-1}} \\
& \mathbf{D}^{(K)=}\left[\begin{array}{lr}
\mathbf{D}^{(K-1)} & \mathbf{0} \\
\mathbf{0} & \mathbf{D}^{(K-1)}
\end{array}\right] \text { with } \mathbf{D}^{(1)}=\operatorname{diag}\{\mathbf{w}\} .
\end{aligned}
$$

The vector $\mathbf{w}$ in (12) contains the first column of $\mathbf{W}^{(1)}$, i.e. the terms $e^{-j \theta_{i}}$, where $\theta_{i}$ is the total phase increment across the $i$ th prototype signal shown in Fig. 4. A slicer selects the element of $\mathbf{y}_{k}^{(K)}$ with the largest magnitude, and thus determines the received bit.

\subsection{IFO Carrier Frequency Offset Correction}

By excluding the noise term in (3), such that

$$
r[n]=s[n] e^{j \Delta \Omega n},
$$

it is apparent that $\angle \mathcal{E}\left\{r[n] s^{*}[n]\right\} \propto \Delta \Omega$, and therefore $\Delta \Omega$ causes a difference in the phase trajectories of the signal computed by the transmitter and the prototype signal assumed by the receiver in computing its filter coefficients. If an initial phase difference of zero is assumed, this anomaly amounts to $\pm \Delta \Omega \cdot N$ radians across a symbol period. This is illustrated in Fig. 5, which depicts the deviation in phase of the received signal relative to that of the matched filter coefficients. In Fig. 5 it is apparent that for the correctly matched filter, or row of $\mathbf{W}^{(1)}$, the phase of its tap outputs will grow in the positive or negative direction, from the first to the last column, for a positive or negative carrier frequency offset respectively.

Thus, in the low-complexity MFB receiver, an indication of the sign and magnitude of the carrier frequency offset $\Delta \Omega$ can

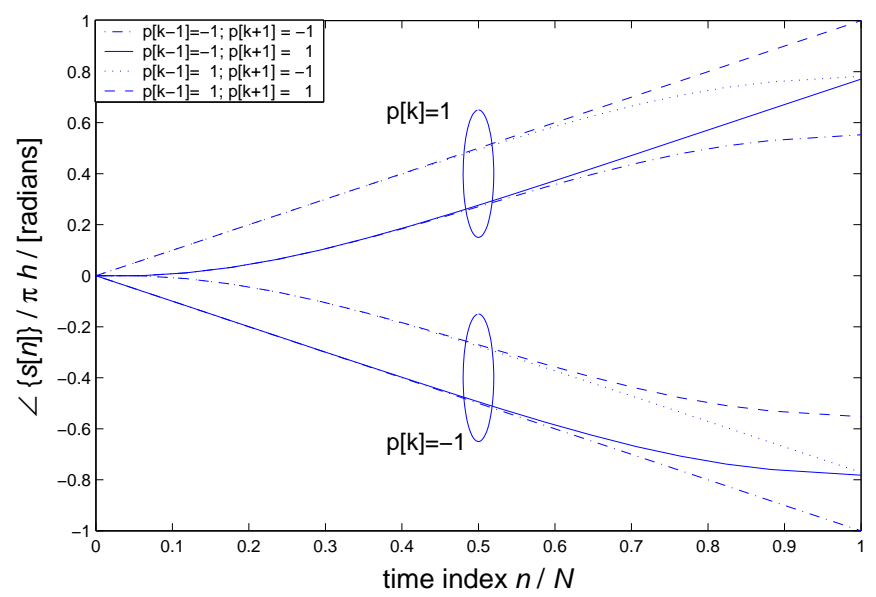

Fig. 4. Example of the legitimate phase trajectories of $s[n]$ for one bit period when $K_{B T}=0.5, L=3, h=0.35$ and an initial phase of zero. 
be obtained via the phase term

$$
\begin{aligned}
\xi_{\Omega, \mathrm{IFO}} & =\angle \mathcal{E}\left\{\left(y_{k}^{(K-(K-1) / 2)} \cdot\left(y_{k}^{(K-(K+1) / 2)}\right)^{*}\right)\right\} \\
& \propto \Delta \Omega-\Delta \hat{\Omega} .
\end{aligned}
$$

Note that in (13), the quantities $y_{k}^{(a)}$ are the intermediate filter outputs after they have been appropriately rotated and accumulated by matrices $\mathbf{D}^{(a)}, \mathbf{A}^{(a)}$, and $\mathbf{M}^{(a)}$ to reflect the phase gained over the preceding $(a-1)$ symbol stages, in other words $y_{k}^{(a)}$ is the element of $\mathbf{y}_{k}^{(a)}$ in Fig. 3 with the largest magnitude. Hence, $\xi_{\Omega \text {,IFO }}$ is a measure of the phase difference between the transmitted signal, and that assumed by the receiver to compute its filter coefficients, during the $\left(k-\frac{K-1}{2}\right)$ th symbol period. It is important to realise that during the $k$ th symbol period, it is the symbol at the center of the current observation interval, or $p\left[k-\frac{K-1}{2}\right]$, that is estimated most accurately, and so fewer frequency tracking errors will be made if the relative phase increment of the received signal, and that of the receiver prototype signal are compared during this period.

The sketch in Fig. 6, in which $K=5$, is meant to support the above explanation. It portrays the phase trajectory of the outputs of filters matched to an arbitrary 5-symbol sequence. It is important to note that for this argument to hold we must consider only the filter that matches the received signal, because in that case, the phase gain in the received sequence due to the modulating baseband signal is cancelled out by the filter coefficients, leaving only phase gain due to parameter offsets. In this case independent of $k$ (13) can be rewritten as [6]

$$
\begin{aligned}
\xi_{\Omega, \mathrm{IFO}}=\angle \mathcal{E}\left\{\left(\sum_{n=(k-K) N+1}^{(k-(K-1) / 2) N} e^{j(\Delta \Omega-\Delta \hat{\Omega}) n}\right) \cdot\right. \\
\left.\cdot\left(\sum_{n=(k-K) N+1}^{(k-(K+1) / 2) N} e^{j(\Delta \Omega-\Delta \hat{\Omega}) n}\right)^{*}\right\} \\
=\angle \mathcal{E}\left\{\left(\sum_{n=1}^{(K+1) N / 2} e^{j(\Delta \Omega-\Delta \hat{\Omega}) n}\right) \cdot\right. \\
=\frac{N}{2}(\Delta \Omega-\Delta \hat{\Omega})
\end{aligned}
$$

where $\Delta \hat{\Omega}$ is the receivers estimate of the transmitters carrier frequency offset. The development in (15) is possible because [14]

$$
\angle\left\{\sum_{n=\alpha}^{\beta} e^{j \theta n}\right\}=\frac{1}{2}(\alpha+\beta) \theta .
$$

From the discussions above, and particularly from (15), it can be asserted that if $\Delta \Omega>\Delta \hat{\Omega}$ then $\xi_{\Omega \text {,IFO }}>0$, and viceversa if $\Delta \Omega<\Delta \hat{\Omega}$. Hence, $\Delta \hat{\Omega}$ can be adjusted according to

$$
\Delta \hat{\Omega}[k+1]=\Delta \hat{\Omega}[k]+\mu_{\Omega} \cdot \hat{\xi}_{\Omega, \mathrm{IFO}}[k]
$$

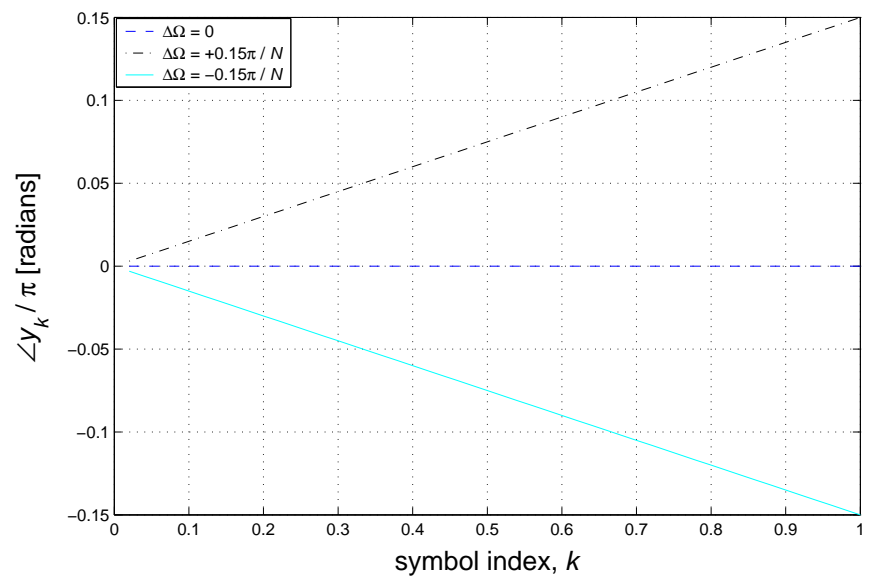

Fig. 5. Relative increment in phase across a symbol period, between a received signal and the corresponding matched filter coefficients, when carrier frequency offsets exist between transmitter and receiver.

where $\hat{\xi}_{\Omega, \mathrm{IFO}}[k]$ is an instantaneous inference of the term in (13) based on a single symbol period, or simply

$$
\hat{\xi}_{\Omega, \mathrm{IFO}}[k]=\angle\left\{y_{k}^{(K-(K-1) / 2)} \cdot\left(y_{k}^{(K-(K+1) / 2)}\right)^{*}\right\}
$$

Note that in (16), the element $y_{k}^{(a)}$ is based on the estimated symbol sequence rather than the true quantities assumed in (13). Since all terms on the right of (16) are available, per interation, from the proposed low-cost MFB, the only additional complexity arises from the $2^{L} N$ MACs necessary to modify $\mathbf{W}^{(1)}$ and consequently $\mathbf{D}^{(a)}$.

\subsection{Modulation Index}

It follows from the development in Sec. 2, but mainly from (1) and (2), that $\angle\{s[n]\} \propto h \cdot p[k]$, and hence the phase tree for the transmitted signal $s[n]$, shown for the first symbol period in Fig. 4, would fan further out for a larger modulation

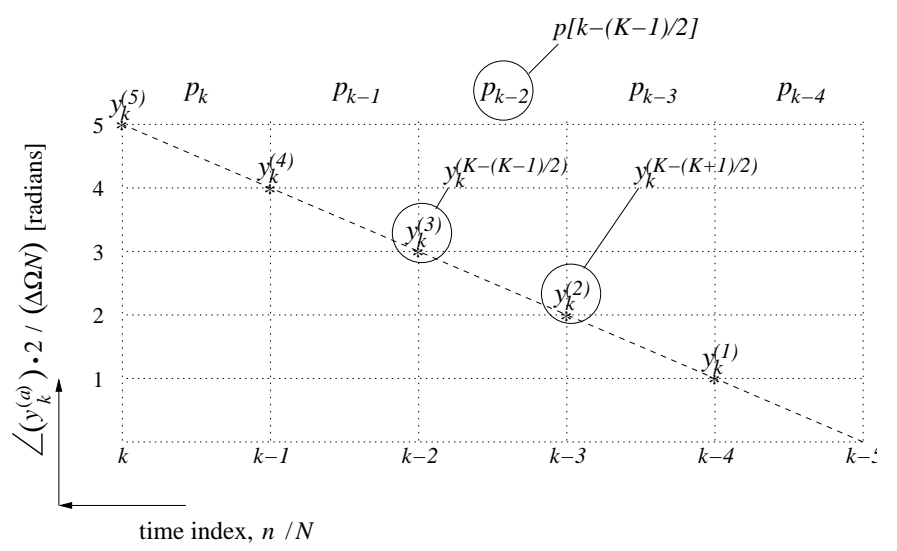

Fig. 6. Sketch of a phase tree of the largest matched filter output $y_{k}^{(a)}$, for $a \in\{1,2,3,4,5\}$ and $K=5$, when a carrier frequency offset $\Delta \Omega$ exists. 

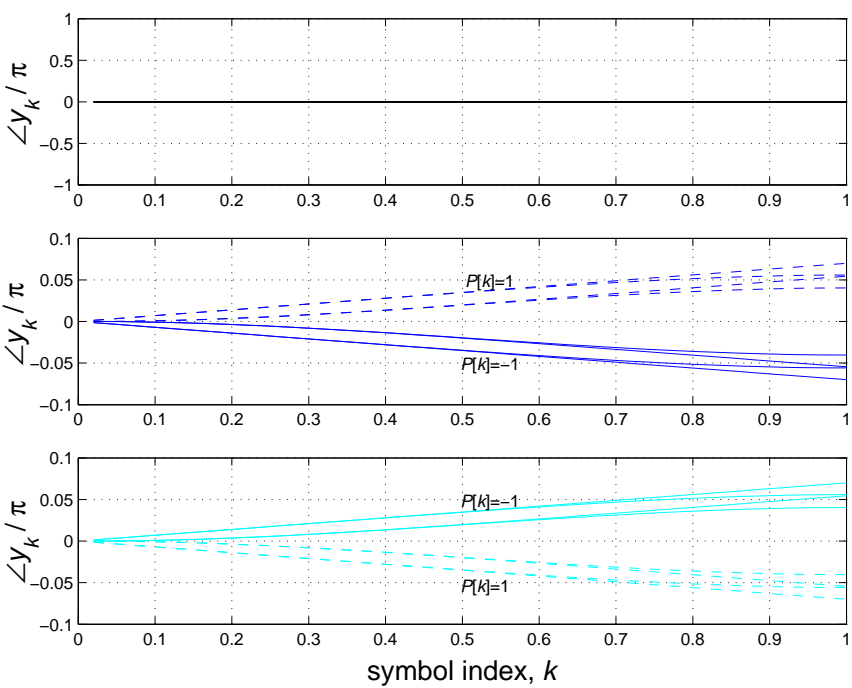

Fig. 7. Relative increment in phase across a symbol period, between a received signal and the corresponding matched filter coefficients, when modulation index offsets of $\Delta h=0$ (top), $\Delta h=0.07$ (middle), and $\Delta h=-0.07$ (bottom) exist between transmitter and receiver.

index. Consequently, if the receiver adopts a modulation index $\hat{h}$, and $h>\hat{h}$, then phase trajectories of the transmitter will be positive with respect to those assumed by the receiver when $p[k]=1$, and negative with respect to the phase trajectories assumed by the receiver when $p[k]=-1$. Accordingly, the deviation in phase increment between the transmit signal and the corresponding matched filter coefficients amounts to approximately $p[k](h-\hat{h}) \pi$ across a symbol period. This discrepancy can be observed from the angle of the output of the correctly matched intermediate filter after it has been appropriately rotated and accumulated to reflect the phase gain over preceding symbol stages. This is exemplarily shown in Fig. 7 for a single symbol, whereby the dashed and solid lines imply trajectories due to a modulating pulse of +1 and -1 respectively. It is clear from Fig. 7 that a negative modulation index offset reflects the phase tree about the zero axis.

Hence, in the low-cost MFB receiver, we employ the phase term

$$
\begin{aligned}
\xi_{h, \mathrm{IFO}}= & \mathcal{E}\left\{\angle\left(y_{k}^{(K-(K-1) / 2)} \cdot\left(y_{k}^{(K-(K+1) / 2)}\right)^{*}\right) .\right. \\
& \left.\cdot p\left[k-\frac{K-1}{2}\right]\right\} \\
\propto & h-\hat{h}
\end{aligned}
$$

to determine the amount by which the transmitter modulation index exceeds that of the receiver. Analogous to Sec. 4.2, in (17) the quantity $y_{k}^{(a)}$ refers to the maximum element of $\mathbf{y}_{k}^{(a)}$ in Fig. 3, associated with the correct symbol sequence, and leading to the detection of the symbol at the center of the observation interval, $p\left[k-\frac{K-1}{2}\right]$. The complex conjugate term in (17) ensures that the phase is measured relative to zero, while as implied in Fig. 7, $p\left[k-\frac{K-1}{2}\right]$ compensates for the sign change im- posed by the modulating symbol during the interval over which signal phase is assessed. This is more obvious for the simple case where an all 1 or all -1 symbol steam is transmitted, and if we assume $K_{B T}=\infty$ so that no ISI exists. Therefore we have

$$
|g[n]|=\frac{1}{2 N},
$$

while

$$
\begin{aligned}
|\omega[n]| & =2 \pi h \cdot|g[n]| \\
& =\frac{\pi h}{N},
\end{aligned}
$$

such that (17) can be derived as follows

$$
\begin{aligned}
& \xi_{h, \mathrm{IFO}} \approx \mathcal{E}\left\{\angle \left(\left(\sum_{\kappa=k-K+1}^{k-(K-1) / 2} \sum_{n=(\kappa-1) N+1}^{\kappa N} e^{j p[\kappa] \pi(h-\hat{h}) n / N}\right) .\right.\right. \\
& \left.\cdot\left(\sum_{\kappa=k-K+1}^{k-(K+1) / 2} \sum_{n=(\kappa-1) N+1}^{\kappa N} e^{-j p[\kappa] \pi(h-\hat{h}) n / N}\right)\right) \text {. } \\
& \text {. } \left.p\left[k-\frac{(K-1)}{2}\right]\right\} \\
& \approx \mathcal{E}\left\{\angle \left(\left(\sum_{n=(k-K) N+1}^{(k-(K-1) / 2) N} e^{j \pi(h-\hat{h}) n / N}\right)\right.\right. \text {. } \\
& \left.\left(\sum_{n=(k-K) N+1}^{(k-(K+1) / 2) N} e^{-j \pi(h-\hat{h}) n / N}\right)\right) \text {. } \\
& \text {. } \left.p\left[k-\frac{(K-1)}{2}\right]\right\} \\
& \approx\left(\left((k N-K N+1)+\left[k N-\left(\frac{K-1}{2}\right) N\right]\right)-\right. \\
& \left.\left((k N-K N+1)+\left[k N-\left(\frac{K+1}{2}\right) N\right]\right)\right) . \\
& \frac{\pi(h-\hat{h})}{2 N} \cdot p\left[k-\frac{(K-1)}{2}\right] \\
& \approx \frac{\pi}{2}(h-\hat{h}) \text {. }
\end{aligned}
$$

From the above discussion, and mainly from (18), it follows that if $h>\hat{h}$ then $\xi_{h \text {,IFO }}>0$, and the converse is true when $h<$ $\hat{h}$. Therefore to adapt the receivers estimate of the modulation index $\hat{h}$, we employ an iterative technique

$$
\hat{h}[k+1]=\hat{h}[k]+\mu_{h} \cdot \hat{\xi}_{h, \mathrm{IFO}}[k]
$$

where $\hat{\xi}_{h, \mathrm{IFO}}[k]$ is an instantaneous value of the term in (17) based on a single symbol period, and evaluated as

$$
\hat{\xi}_{h, \mathrm{IFO}}[k]=\angle\left\{y_{k}^{(K-(K-1) / 2} \cdot\left(y_{k}^{(K-(K+1) / 2)}\right)^{*}\right\} \cdot \hat{p}[k]
$$

Bear in mind that the element $y_{k}^{(a)}$ in (20), is based on the estimated symbol sequence and the detected symbol $\hat{p}[k]$, rather than the true quantities assumed in (17), and that ideally $\hat{p}[k]=$ $p\left[k-\frac{K-1}{2}\right]$. 
The adoption of $\hat{h}[k+1]$ following (19) requires few extra computations because the coefficients in $\mathbf{W}^{(1)}$ and subsequently $\mathbf{D}^{(a)}$ can be recalculated at the same time as the carrier frequency offset.

\section{Results}

In our experiments $K_{B T}=0.5, h=0.35$ and $N=2$ in order to simulate a Bluetooth system. The efficacy of the SG and IFO algorithms is evident from the learning curves in Fig. 8, which demonstrates that if an equal step size is employed the IFO algorithms will have a greater convergence speed.

However, after convergence the SG carrier frequency offset correction algorithm and the IFO carrier frequency and modulation index offset correction algorithms are able to restore

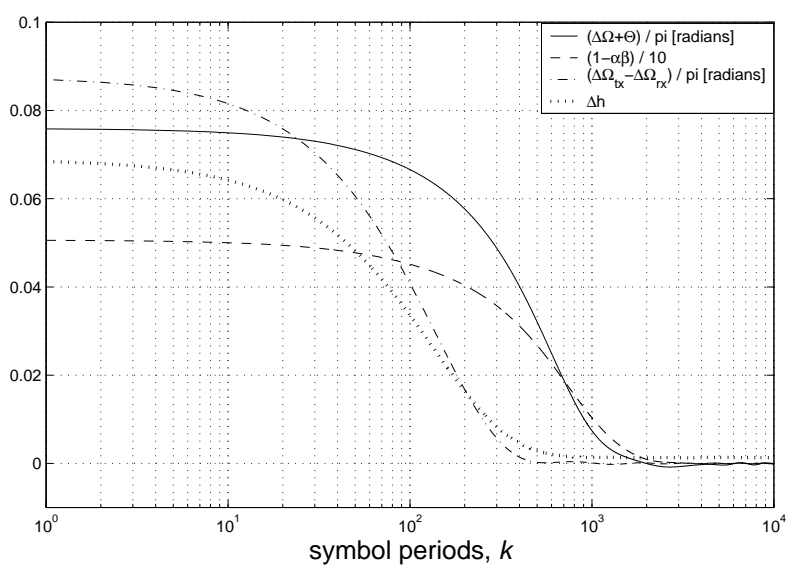

Fig. 8. Learning curves for the SG and IFO carrier frequency and modulation index offset correction algorithms with $K_{B T}=0.5, h=0.35, N=2, \Delta \Omega=0.075 \pi, \mu_{\Theta}=\mu_{B}=$ $\mu_{\Omega}=\mu_{h}=0.005$.

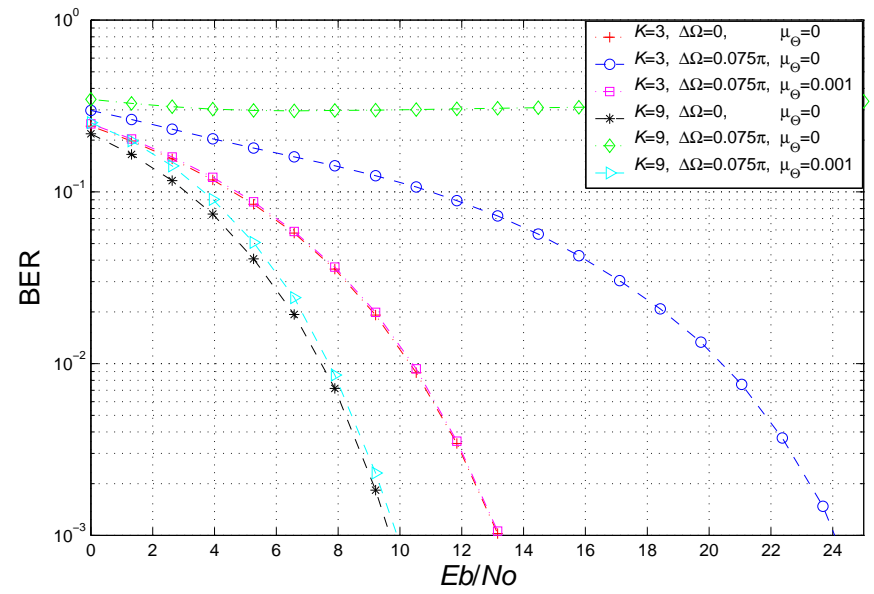

Fig. 9. BER performance in with a carrier frequency offset and correction by the SG tracking algorithm, using $N=2$, $K_{B T}=0.5, h=0.35$, and $M=1$.

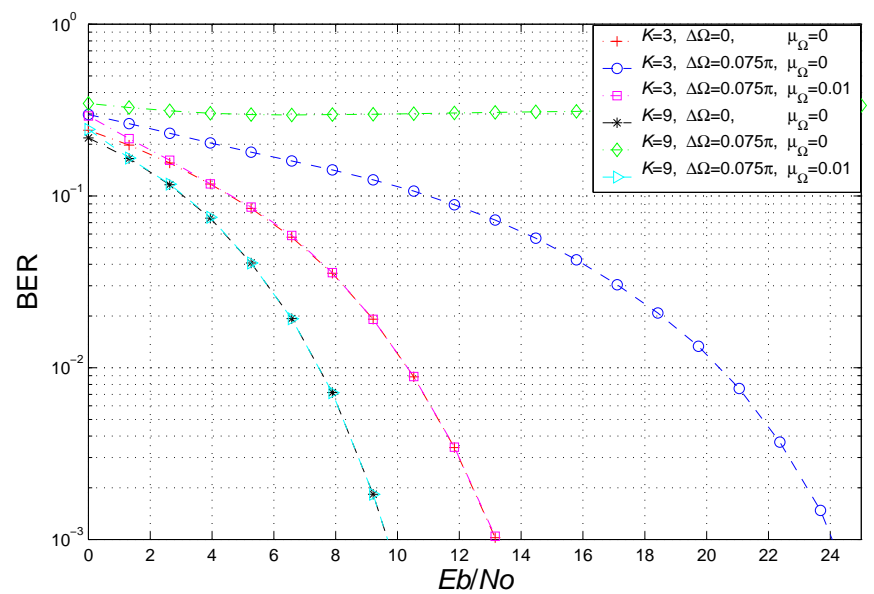

Fig. 10. BER performance in with a carrier frequency offset and correction by the IFO tracking algorithm, using $N=2$, $K_{B T}=0.5$, and $h=0.35$.

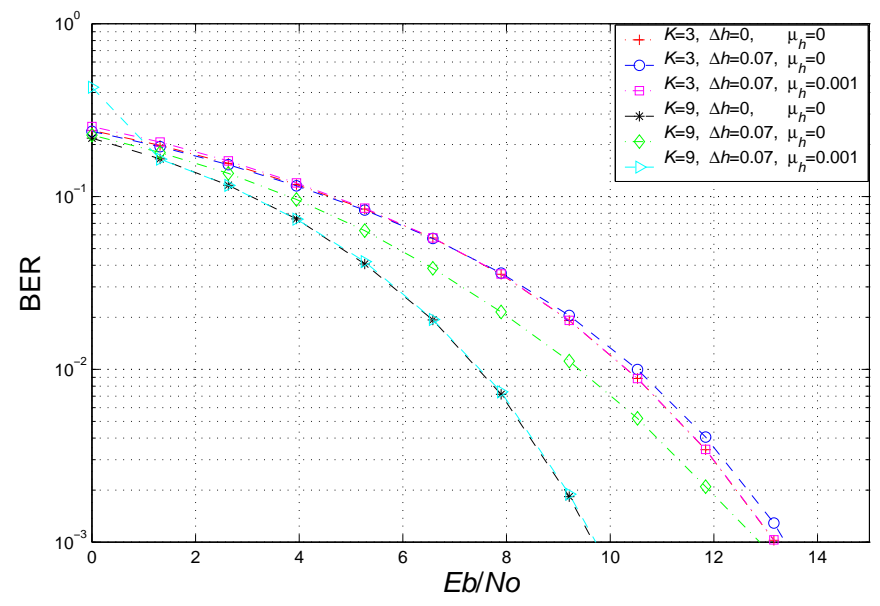

Fig. 11. BER performance in with a modulation index offset and correction by the IFO tracking algorithm, using $N=2$, $K_{B T}=0.5$, and $h=0.35$.

the BER performance to that obtained in AWGN. This is exemplified in the BER results depicted in Figs. 9, 10, and 11, where it is evident that the system is saved from collapse when $K=9$ and the maximum permissible carrier frequency offset for Bluetooth exists, while $11 \mathrm{~dB}$ less is required to achieve the minimum acceptable Bluetooth performance of $B E R=10^{-3}$. On the other hand, deploying the IFO modulation index offset correction procedure will save $3.5 \mathrm{~dB}$ and $0.5 \mathrm{~dB}$ for $K=9$ and $K=3$ respectively.

\section{Conclusion}

Carrier frequency and modulation index offsets can cause severe loss in performance in Bluetooth receivers. This is especially the case with long filter lengths $K$ over which such errors accumulate. However, longer observation intervals actually have better performance in synchronised conditions. Employing the algorithms described above alleviate can alleviate 
the dilemma in choosing $K$ by providing some assurance that transmitter and receiver carrier frequency and modulation index can be synchronised online.

\section{References}

[1] William P. Osborne and Micheal B. Luntz, "Coherent and Noncoherent Detection of CPFSK," in IEEE Transactions on Communications, August 1974, vol. COM-22, pp. 1023-1036.

[2] John B. Anderson, Tor Aulin, and Carl-Erik Sundberg, Digital Phase Modulation, Plenum Press, New York and London, 1986.

[3] John G. Proakis, Digital Communications, McGraw-Hill, New York, 3rd edition, 1995.

[4] Charles Tibenderana and Stephan Weiss, "LowComplexity High-Performance GFSK Receiver With Carrier Frequency Offset Correction," in Proc. IEEE International Conference on Acoustics, Speech, and Signal Processing, Montreal, Canada, May 2004, vol. IV, pp. 933-936.

[5] Charles Tibenderana and Stephan Weiss, "Fast Multilevel GFSK Matched Filter Receiver," in Proc. Institute of Mathematics and its Applications Conference on Mathematics in Signal Processing, Cirencester, Essex, UK, December 2004, pp. 191-194.

[6] Charles Tibenderana, "A High-Performance, Efficient, and Reliable Receiver for Bluetooth Signals," PhD Thesis, University of Southampton, submitter August 2005.

[7] Charles Tibenderana and Stephan Weiss, "Rapid Equalisation for a High Integrity Bluetooth Receiver," in Proc. IEEE Workshop on Statistical Signal Processing, Bordeaux, France, July 2005, Submitted to.

[8] Bluetooth Special Interest Group, Specification of the Bluetooth System, February 2002, Core.

[9] Craig Robinson and Alan Purvis, "Demodulation of Bluetooth GFSK Signals Under Carrier Frequency Error Conditions," in Proc. IEE Colloquium on DSP Enabled Radio, Livingston, Scotland, UK, September 2003.

[10] Amir Soltanian and Robert E. Van Dyck, "Performance of the Bluetooth System in Fading Dispersive Channels and Interference," in Proc. Global Telecommunications Conference, San Antonio, Texas, November 2001, vol. 6, pp. 3499-3503.

[11] Bernard Widrow and Samuel D. Stearns, Adaptive Signal Processing, Prentice Hall, Inc, New Jersey, 1985.

[12] Charles Tibenderana and Stephan Weiss, "Blind Equalisation and Carrier Offset Compensation for Bluetooth Signals," in Proc. European Signal Processing Conference, Vienna, Austria, September 2004, pp. 909-912.
[13] Charles Tibenderana and Stephan Weiss, "A Low-Cost Scalable Matched Filter Bank Receiver for GFSK Signals with Carrier Frequency and Modulation Index Offset Compensation," in Proc. Asilomar Conference on Signals, Systems, and Computers, Pacific Groove, California, USA, November 2004, pp. 682-686.

[14] John G. Proakis and Dimitris G. Manolakis, Digital Signal Processing, Prentice Hall, Upper Saddle River, New Jersey, 3rd edition, 1996. 